\title{
Knowledge of Human Papillomavirus Infection, Cervical Cancer and Willingness to pay for Cervical Cancer Vaccination among Ethnically Diverse Medical Students in Malaysia
}

\author{
Mari Kannan Maharajan ${ }^{1 *}$, Kingston Rajiah $^{1}$, Kelly Sze Fang Num ${ }^{2}$, Ng Jin Yong $^{3}$
}

\begin{abstract}
The primary objective of this study was to assess the knowledge of medical students and determine variation between different cultural groups. A secondary aim was to find out the willingness to pay for cervical cancer vaccination and the relationships between knowledge and attitudes towards Human Papillomavirus vaccination. A cross-sectional survey was conducted in a private medical university between June 2014 and November 2014 using a convenient sampling method. A total of 305 respondents were recruited and interviewed with standard questionnaires for assessment of knowledge, attitudes and practice towards human papilloma virus and their willingness to pay for HPV vaccination. Knowledge regarding human papilloma virus, human papilloma virus vaccination, cervical cancer screening and cervical cancer risk factors was good. Across the sample, a majority $\mathbf{9 0 \%}$ ) of the pupils demonstrated a high degree of knowledge about cervical cancer and its vaccination. There were no significant differences between ethnicity and the participants' overall knowledge of HPV infection, Pap smear and cervical cancer vaccination. Some $88 \%$ of participants answered that HPV vaccine can prevent cervical cancer, while $81.5 \%$ of medical students said they would recommend HPV vaccination to the public although fewer expressed an intention to receive vaccination for themselves .
\end{abstract}

Keywords: HPV vaccine - cervical cancer - knowledge - attitude - willingness to pay - medical students - Malaysia

Asian Pac J Cancer Prev, 16 (14), 5733-5739

\section{Introduction}

Cervical cancer is an important public health concern worldwide, and especially in developing countries. In Malaysia, cervical cancer was the second most common cancer in women after breast cancer and colorectal cancer (Kwang et al., 2014). The connection between cervical cancer and oncogenic human papillomavirus (HPV) infection has been well-known. Virtually oncogenic HPV DNA involved in all cervical cancers (Uzunlar et al., 2013). HPV type 16 and HPV type 18 are accounted for about $70 \%$ of cervical cancer cases globally (David, 2008). These types of HPV also cause penile cancer in men (Hoque et al., 2009) whereas HPV type 6 and 11 cause genital warts in both men and women (La Torre et al., 2007). Prophylactic HPV vaccines have been developed to prevent HPV-related cervical cancer (Ezem, 2007). There are two different vaccines: the first one is a bivalent vaccine, which offers protection against HPV type 16 and 18 and the second one is tetravalent which offers protection against HPV type 6, 11, 16 and 18 (Aljunid, 2010).
Previous studies on the acceptability of the HPV vaccination for cervical cancer prevention showed that the rate of acceptability varies among studied populations (McClelland and Liamputtong, 2006; Hopenhayn et al., 2007; Wen et al., 2014). Earlier studies mentioned that there are few factors such as lack of information regarding cervical cancer and HPV vaccination (Hopenhayn et al., 2007), safety and efficacy of HPV vaccination (McClelland and Liamputtong, 2006), high cost (McClelland and Liamputtong, 2006) willingness to pay for vaccination and embarrassment of getting a vaccination for a sexually transmitted disease (Wen et al., 2014) were the causes for inadequate acceptance of voluntary vaccination for HPV infection. In Malaysia, national Pap smear screening programme is available for free of charge for women attending public health facilities. However, the utilisation of Pap smear programme in the past is only about $40 \%$ (National Health Morbidity Survey III, 2008).

The healthcare professionals play an instrumental part in creating awareness of cervical cancer and facilitating HPV vaccination among public and have the responsibilities to address the barriers to prevent

${ }^{1}$ Department of Pharmacy Practice, School of Pharmacy International Medical University, ${ }^{2}$ Department of Nutrition \& Dietetics School of Health Sciences, International Medical University, ${ }^{3}$ School of Pharmacy International Medical University, Malaysia, Kuala Lumpur, Malaysia*For correspondence: marikannan@imu.edu.my 
cervical cancer. The effectiveness of HPV vaccination will basically depend on how health professionals have been oriented and their willingness to indicate HPV vaccination to the public. University students represent the risk group who are open to negative health behaviors and effects of negative environmental factors (Yilmazel and Duman, 2014). Being an easily accessible group, their knowledge of HPV infection and their attitude towards cervical cancer vaccination could provide insight into the willingness to promote vaccines by the future practitioners to the general population in Malaysia. It is indispensable to distinguish if the students of medical schools, future health care pros, have accurate knowledge regarding HPV vaccination, so that they can provide reliable information to the public (Wen et al., 2014). Malaysia is a multi-ethnic and multireligious society and educating the population about HPV infection and promoting cervical cancer vaccination remains a great challenge for the health care professionals (Wong LP, 2011).

Hence, it is important to assess current set of medical students' knowledge about HPV infections and their attitude towards cervical cancer vaccinations as they will serve the community in the future. The main aim of this work was to evaluate the knowledge of medical students and find out the differences of their knowledge level between different ethnic groups. The secondary purpose of this work was to find out the willingness to pay for cervical cancer vaccination and relationship between their knowledge and attitude towards HPV vaccination.

\section{Materials and Methods}

\section{Study participants}

A cross-sectional study was conducted among the medical students from the Faculty of Medicine in one of the private Universities in Malaysia. This was a six month study carried out between June and November 2014. The participants were explained about the study and written consent was received before the questionnaire was made away. Inclusion criteria for this study merited being a student in the medical program. Participation in the study was voluntary and anonymous.

\section{Sampling technique}

Convenient sampling method was used (Saravanan and Kingston, 2014). The sample size required for this study was calculated using a RAOSOFT calculator with a $5 \%$ margin of error and $95 \%$ confidence level. Agreeing to this calculation, 273 students were needed from the faculty of medicine for this work. Withal, 302 students participated in this work, which was more than sufficient for this study.

\section{Study instrument}

Anonymous, self-administered questionnaires were prepared which contained a mix of 5-point Likert scale, dichotomous (yes/no), and free text completion items. The questionnaire composed of four parts. The first part concerned about participants' demographic data. The second part was about participants' personal life style. The third part contained questions that measured the participants' knowledge of HPV infection, cervical cancer and Pap smear test and their attitude towards HPV vaccination. The fourth part measured the participants' willingness to pay for HPV vaccination. The knowledge level was categorised with a range as low (0-5), moderate (6-11) and high (12-17) whereas the attitude level was categorised as negative (0-2.33), neutral (2.34-3.67) and positive (3.68-5.00).

\section{Reliability and Validity of questionnaire}

A questionnaire was validated for reliability of internal consistency, by conducting a pilot study with 20 participants. The internal consistency of the 13-item knowledge of HPV and genital warts questions and 4-item knowledge of Pap-smear test were evaluated by Cronbach's alpha. Founded on these determinations from the pilot testing, minor modification on the questionnaire was constructed. The information from pilot testing was not admitted in the last analysis. The questionnaire was face validated by content experts.

\section{Data analysis}

Data analysis was done using the SPSS software version 22.0. Both descriptive and univariate analyses were done to find out the population characteristics, knowledge of HPV infection, cervical cancer and Pap smear test and their attitude towards cervical cancer vaccination. They provide simple summaries about the sample and the measures. All categorical variables including respondents' socio-demographic characteristics, cultural differences in participant's knowledge of HPV infection, Pap smear and cervical cancer vaccination and other attitudinal items are expressed as frequencies and percentages. The willingness to pay was presented as frequencies.

\section{Results}

To the 305 distributed, 302 completed questionnaires were given back, implying a response rate of $99 \%$. The sample's age range was 20-26 years (mean 23.5 years, $\mathrm{SD} \pm 1.0)$. The majority (74.2\%) declared their ethnicity as Chinese. None had children and was married, although $56.2 \%$ reported as being in a relationship. All the participants were studying for an undergrad degree. To the highest degree, $86 \%$ declared they had never smoked cigarettes and $14 \%$ reported themselves to be smokers (Table 1).

Students' knowledge about cervical cancer was assessed by using questions to which they had to choose the answer "true", "false", or "don't know". The knowledge score was computed with one point given for each correct answer to these questions. The mean score for knowledge of HPV, cervical cancer and Pap smear test were 12.2 out of a maximum score of $17 \pm 2.54$. The majority $(93.7 \% \pm$ 1.42) were sure that HPV is related to cervical cancer. The Cronbach's alpha value was 0.873 . Table 2 showed the percentage of correct responses to the questions probing knowledge about HPV infection, Pap-smears and the HPV vaccination within various ethnics of the participants. All the students were aware of cervical cancer, HPV infection and HPV vaccine. Majority (90\%) of the students had 
Knowledge of HPV Infection, Cervical Cancer and Willingness to Pay for Vaccination among Medical Students in Malaysia

a high level of knowledge. Overall, only $93.7 \%$ of the participants knew that persistent genital HPV infection causes cervical cancer. More than $90 \%$ of the participants know that HPV infection can induce cervical cancer and

Table 1. Demographic Characteristics of Participants

\begin{tabular}{llr}
\hline Characteristic & & $\mathrm{N}=302$ \\
\hline Gender & Male & $139(46.0 \%)$ \\
& Female & $163(54.0 \%)$ \\
Ethnicity & Malay & $34(11.3 \%)$ \\
& Chinese & $224(74.2 \%)$ \\
& Indian & $32(10.6 \%)$ \\
& Others & $12(4.0 \%)$ \\
Religion & Islam & $50(12.6 \%)$ \\
& Buddhist & $127(42.1 \%)$ \\
& Hindu & $37(11.9 \%)$ \\
& Christian & $68(22.5 \%)$ \\
Marital Status & Others & $20(10.6 \%)$ \\
& Married & $0(0 \%)$ \\
Relationship status & Single & $302(100 \%)$ \\
& Not in a relationship & $132(43.8 \%)$ \\
Social habits & In a relationship & $170(56.2 \%)$ \\
& Smokers & $42(14 \%)$ \\
HPV vaccination & Non smokers & $260(86 \%)$ \\
& Received at least once & $42(13.9 \%)$ \\
& Not yet received & $260(86.1 \%)$ \\
\hline
\end{tabular}

it is preventable. Most of the participant $(90.1 \%)$ knew that Pap smear can screen cervical cancer. However, only about half of the participants knew that Pap smear should be done every 3 years and it can be done after the age of 21 . There were no significant differences between ethnicity and the participants' overall knowledge of HPV infection, Pap smear and cervical cancer vaccination. Nevertheless, there were substantial differences noted for the statements "HPV may infect both, adult males and woman" and " $H P V$ can cause other anogenital cancers (eg. penis, anus)". For both these statements, Malay ethnic group students had nominal knowledge compared to other students. However, more than half of the students belongs to Malay ethnic group knew that the HPV vaccine prevents cervical cancer. More than $50 \%$ of Chinese ethnic group students knew HPV is a sexually transmitted disease. Across the sample, the mean total knowledge score was $12.24 \pm 2$ out of 17 .

To assess attitudes towards cervical cancer vaccination in general, all participants $(\mathrm{n}=302)$ were asked to "agree" or "disagree" or indicate "don't know" with twelve statements. One point was awarded for each favourable response, so a score of twelve indicated strong support for cervical cancer vaccination. Table 3 shows participants' attitude towards cervical cancer and HPV vaccination. Data revealed that the overall attitude of participants

Table 2. Participants' Knowledge of HPV Infection, Pap smear and HPV Vaccination

\begin{tabular}{|c|c|c|c|c|c|c|c|}
\hline & \multirow{2}{*}{$\frac{\text { Correct answer }}{\mathrm{n},(\text { frequency) }}$} & \multirow{2}{*}{$\frac{\text { Incorrect answer }}{\mathrm{n},(\text { frequency) }}$} & \multicolumn{5}{|c|}{ Percentage of correct answers within various ethnicity } \\
\hline & & & Malay & Chinese & Indian & others & P-value \\
\hline \multicolumn{8}{|l|}{ HPV can cause cervical cancer } \\
\hline & $283(93.7 \%)$ & $19(6.3 \%)$ & 22.1 & 29.5 & 23.8 & 24.6 & 0.488 \\
\hline \multicolumn{8}{|l|}{ HPV infections are preventable } \\
\hline & $289(95.7 \%)$ & $13(4.3 \%)$ & 23.4 & 22.5 & 27.8 & 26.3 & 0.311 \\
\hline \multicolumn{8}{|c|}{ Condom use can prevent HPV infection } \\
\hline & $235(77.8 \%)$ & $67(12.2 \%)$ & 28.6 & 26.3 & 20.8 & 24.3 & 0.652 \\
\hline \multicolumn{8}{|c|}{ HPV is a sexually transmitted disease (STD) } \\
\hline & $251(83.1 \%)$ & $51(16.9 \%)$ & 22.9 & 50.6 & 21.3 & 6.2 & 0.421 \\
\hline \multicolumn{8}{|l|}{ HPV infection is frequent } \\
\hline & $196(64.9 \%)$ & $106(35.1 \%)$ & 32.2 & 6.3 & 25.4 & 36.1 & 0.205 \\
\hline \multicolumn{8}{|l|}{ HPV infection can last for years } \\
\hline & $237(78.5 \%)$ & $65(21.5 \%)$ & 27.8 & 23.4 & 22.5 & 26.3 & 0.805 \\
\hline \multicolumn{8}{|c|}{ Cervical cancer is caused by persistent HPV infection } \\
\hline & $240(79.5 \%)$ & $63(20.5 \%)$ & 24.3 & 28.6 & 20.8 & 26.3 & 0.461 \\
\hline \multicolumn{8}{|c|}{ HPV may infect both, men and woman } \\
\hline & $237(78.5 \%)$ & $65(21.5 \%)$ & 12.5 & 22.9 & 36.4 & 28.2 & 0.001 \\
\hline \multicolumn{8}{|c|}{ Most HPV infection resolves spontaneously } \\
\hline & $88(29.1 \%)$ & $214(70.9 \%)$ & 24.6 & 23.8 & 29.5 & 22.1 & 0.903 \\
\hline \multicolumn{8}{|c|}{ HPV can infect you without symptoms } \\
\hline & $234(77.5 \%)$ & $68(22.5 \%)$ & 27.8 & 22.5 & 23.4 & 26.3 & 0.478 \\
\hline \multicolumn{8}{|l|}{ HPV can cause genital warts } \\
\hline & $265(87.7 \%)$ & $37(22.3 \%)$ & 28.6 & 24.3 & 20.8 & 26.3 & 0.211 \\
\hline \multicolumn{8}{|c|}{ HPV can cause other anogenital cancers (eg. penis, anus) } \\
\hline & $192(63.6 \%)$ & $110(36.4 \%)$ & 11.8 & 35.1 & 29.2 & 23.9 & 0.032 \\
\hline \multicolumn{8}{|c|}{ HPV vaccine prevents around $70 \%$ of cervical cancer } \\
\hline & $210(69.5 \%)$ & $92(30.5 \%)$ & 50.6 & 21.3 & 22.9 & 6.2 & 0.658 \\
\hline \multicolumn{8}{|c|}{ Pap Smear can screen cervical cancer } \\
\hline & $272(90.1 \%)$ & $30(8.9 \%)$ & 32.2 & 25.4 & 6.3 & 36.1 & 0.401 \\
\hline \multicolumn{8}{|c|}{ Pap Smear is very or relatively effective in screening cervical cancer } \\
\hline & $242(80.1 \%)$ & $60(19.9 \%)$ & 22.5 & 23.4 & 27.8 & 26.3 & 0.105 \\
\hline \multicolumn{8}{|c|}{ Pap Smear should be done every 3 years } \\
\hline & $155(51.3 \%)$ & $147(48.7 \%)$ & 24.3 & 28.6 & 20.8 & 26.3 & 0.705 \\
\hline \multicolumn{8}{|c|}{ Pap Smear can be done after the age of 21} \\
\hline & $150(49.7 \%)$ & $152(50.3 \%)$ & 28.2 & 36.4 & 22.9 & 12.5 & 0.161 \\
\hline
\end{tabular}


Table 3. Participants' Attitude Towards Cervical Cancer and HPV Vaccination

\begin{tabular}{|c|c|c|c|c|c|c|c|}
\hline \multirow{2}{*}{$\frac{\text { Disagree }}{\mathrm{n},(\text { frequency })}$} & \multirow{2}{*}{$\frac{\text { Not sure }}{n,(\text { frequency) }}$} & \multirow{2}{*}{$\frac{\text { Agree }}{n,(\text { frequency) }}$} & \multicolumn{5}{|c|}{ Percentage of agreement within various religions } \\
\hline & & & Malay & Chinese & Indian & others & P-value \\
\hline \multicolumn{8}{|c|}{ Cervical cancer is a severe disease. } \\
\hline $13(4.3 \%)$ & $9(3.0 \%)$ & $280(92.7 \%)$ & 22.5 & 26.3 & 27.8 & 23.4 & 0.805 \\
\hline \multicolumn{8}{|c|}{ Cervical cancer is preventable. } \\
\hline $19(6.3 \%)$ & $22(7.3 \%)$ & $261(86.5 \%)$ & 20.8 & 28.6 & 24.3 & 26.3 & 0.461 \\
\hline \multicolumn{8}{|c|}{ I am susceptible to HPV infection. } \\
\hline $95(31.5 \%)$ & $68(22.5 \%)$ & $139(46 \%)$ & 28.2 & 22.9 & 36.4 & 12.5 & 0.903 \\
\hline \multicolumn{8}{|c|}{ HPV vaccine is helpful to prevent cervical cancer. } \\
\hline $11(3.7 \%)$ & $25(8.3 \%)$ & $265(88 \%)$ & 19.6 & 27.1 & 29.5 & 23.8 & $0.003 * *$ \\
\hline \multicolumn{8}{|l|}{ HPV vaccine is safe. } \\
\hline \multicolumn{8}{|c|}{ There is less risk involved in being vaccinated than in having HPV infection. } \\
\hline $9(3.0 \%)$ & $33(10.9 \%)$ & $260(86.1 \%)$ & 26.3 & 24.3 & 28.6 & 20.8 & 0.21 \\
\hline \multicolumn{8}{|c|}{ HPV vaccination will not lead to complicated sexual activities. } \\
\hline $34(11.3 \%)$ & $77(25.5 \%)$ & $191(63.2 \%)$ & 30.2 & 35.1 & 12.8 & 23.9 & $0.024 *$ \\
\hline \multicolumn{8}{|c|}{ Vaccinating young people against HPV would not encourage them to become sexually active. } \\
\hline $77(25.5 \%)$ & $87(28.8 \%)$ & $138(73.8 \%)$ & 55.6 & 21.3 & 19.9 & 4.2 & 0.658 \\
\hline \multicolumn{8}{|c|}{ I would not want to be infected with HPV. } \\
\hline $17(5.6 \%)$ & $6(2.0 \%)$ & $279(92.4 \%)$ & 22.2 & 25.4 & 26.3 & 26.1 & 0.401 \\
\hline \multicolumn{8}{|c|}{ Information on HPV helps me to decide whether I should be vaccinated against HPV. } \\
\hline $10(3.3 \%)$ & $18(6.0 \%)$ & $274(90.8 \%)$ & 22.5 & 23.4 & 27.8 & 26.3 & 0.105 \\
\hline \multicolumn{8}{|c|}{ If my doctor thinks HPV vaccination is a good idea, I would vaccinate against HPV. } \\
\hline $14(4.6 \%)$ & $29(9.6 \%)$ & $259(85.8 \%)$ & 24.3 & 28.6 & 20.8 & 26.3 & 0.705 \\
\hline \multicolumn{8}{|c|}{ I would vaccinate against HPV if the vaccination is readily available. } \\
\hline $9(3.0 \%)$ & $22(7.3 \%)$ & $271(89.7 \%)$ & 28.2 & 36.4 & 22.9 & 12.5 & 0.161 \\
\hline
\end{tabular}

towards cervical cancer vaccination was positive. There were no significant differences between ethnicity and the participants' overall attitude towards cervical cancer vaccination. However, there were significant differences observed for the statements " $H P V$ vaccination will not lead to complicated sexual activities" and "HPV vaccine is helpful to prevent cervical cancer". Very few Malay ethnic group students believe that HPV vaccine can prevent cervical cancer. In the same direction, only a few Indian ethnic group students believe that HPV vaccination will not contribute to complicated sexual activities. The higher percentage of Chinese ethnic group students will get their children vaccinated against HPV if the vaccination is readily available. Most of the Malay ethnic group students agreed that vaccinating young people against HPV would not encourage them to become sexually active. Overall, a remarkably higher proportion of participants were aware of cervical cancer vaccination, irrespective of their ethnic group differences. Some of these subjects may not choose a vaccine under any conditions because of religious considerations or opposition to vaccines in general. However, we have no data to identify motives for those who rejected all scenarios.

Several questions concerned the price of the HPV vaccine and the willingness to pay for cervical cancer vaccination were asked to the study population. Based on the answers provided, if it is free of charge, $89.7 \%$ of all participants would request it, but if they had to pay for it, the number remarkably decreased (12.25\%). $89.7 \%$ of survey population predicted the vaccine cost approximately less than Ringgit Malaysia (RM) 200, which is approximately $66 \%$ of the real price (appr. 300 in 2014). $30 \%$ of the participants affirmed that they could not afford the total cost of the three doses of HPV vaccine.
Most of the participants were willing to pay an amount up to RM 500 to receive HPV vaccination. It was reported that some of the medical students were not willing to pay for the HPV vaccines as they reckon the government should take the responsibilities to pay for the vaccines. $12.25 \%$ of respondents mentioned that they cannot afford the expenditure on the vaccines.

\section{Discussion}

Our study identifies similarities and differences in HPV-related knowledge and attitudes towards cervical cancer vaccination and their willingness to pay for HPV vaccines. Earlier research in this area has been conducted without differentiating ethnic groups, addressing barriers specific to subgroups of populations may help to achieve high penetrance of HPV vaccination. The data revealed that the knowledge of medical students on HPV infection, Pap smear and HPV vaccination was high in all three different ethnic groups of students. This finding was consistent with another studies conducted in Malaysia (Ezat et al., 2013; Shafei et al., 2014) and a study from India which reported that $75 \%$ of the participants were willing to get vaccinated (Saha et al., 2010). However, this was contradicting with Al-Dubai et al where the respondents showed lesser level of knowledge of HPV vaccination (Al-Dubai et al., 2013). This result reflected that the medical students in both private and public universities in Malaysia have good knowledge about HPV infections and cervical cancer vaccination.

More than $80 \%$ of medical students are aware that HPV can cause genital warts and cervical cancer. This result is higher than a study conducted on students of University of Saskatchewan Student Health Services, which showed that 
only $33.8 \%$ of students are aware that HPV causes genital warts and $39.0 \%$ knew that HPV can cause cervical cancer (Giede et al., 2010). The other studies conducted on adults (Holcomb 2004) and health care professionals (Songthap, 2009) were also showed higher level of awareness of HPV vaccination. Provision of information about HPV infection and its link to cervical cancer is essential, to enhance HPV vaccination acceptability and eliminating stigma associated with cervical cancer risks (Hoque et al., 2013). Future health professionals need to focus on effectively providing information about the rationale for vaccination in early adolescence such as explaining about the safety of HPV vaccines. Therefore, the knowledge of the medical students is a vital component in promoting HPV vaccination. The availability of the HPV vaccine in Malaysia for a longer duration (more than 4 years) and the clinical exposure of medical students are the major reasons for their higher level of knowledge. Television and newspapers were reported as a major source of information, followed by hospital /health care providers (Kruiroongroj et al., 2014).

Medical students' knowledge is an important parameter for educating the public. The results showed that medical students received more knowledge about Pap smears and cervical cancer than about HPV infection and the HPV vaccine, because cervical cancer topics are available in the medical education syllabus for various years. Parents' feelings, general recommendations, safety, and low cost were reported as the factors influencing HPV vaccine acceptance (Boehner 2003; Chow et al., 2010). Jones and Cook, reported that the acceptance of HPV vaccination was positively affected by health policies encouraging vaccination and revealed the pivotal role of healthcare providers in educating individuals about HPV (Jones and Cook, 2008).

In the present study, $88 \%$ of participants feel that HPV vaccination can prevent cervical cancer. Though $13.9 \%$ of the participants in this survey have received HPV vaccination, only $20.5 \%$ among them have the intention to be immunized. This shows that some received vaccination without their own interest. A study by Madhivanan et al., reported that parents play a major role in vaccinating their daughters against HPV (Madhivanan et al., 2014). In the absence of parent-daughter communication, physicians can play a pivotal role in educating and opening discussions around cervical cancer and other sexually transmitted disease. This is one of the challenges in HPV vaccine delivery in a country with multiethnic populations (Wong, 2009).

The general population is unwilling to receive the vaccination due to the safety issue, embarrassment of taking a sexually transmitted infection vaccine and perception of not having the risk of acquiring HPV infection (Wong, 2011). These factors can be considered as challenges for the future medical practitioners. Kilic et al. (2012) recommended providing sufficient and correct information about the vaccine, for adolescent girls and their parents is important in creating awareness of HPV vaccination. In the present work, $81.5 \%$ of medical students said they will recommend HPV vaccination to public yet though their intention to receive vaccination for themselves is less. However, a higher percentage (87.4\%) of willingness to recommend was shown by an earlier study from a public university in Malaysia (Rashwan et al., 2012). This may be due to the fact that education and awareness programs were organized for public university students to provide them with correct information about the risk of getting cervical cancer and the effective measures to prevent it (Rashwan et al., 2012).

Economic factors were always one of the blockades to make HPV vaccination a successful campaign (Kahn et al., 2009). The participants are willing to pay RM 1197.68 as an average for 3 complete doses of HPV vaccines, which is higher than the actual cost in Malaysia. This finding is consistent with previous studies, which showed a higher estimated median regression-adjusted willingness to pay for HPV vaccination (Liao et al., 2009; Khoo et al., 2011). Correct vaccine knowledge can increase willingness to pay for vaccination. These results highlight the importance of knowledge (Pulcini et al., 2013) and effective interventions to encourage the public to take vaccination against HPV infection. Previous study by Ezat et al., showed that willingness to have HPV vaccination correlated well with the level of knowledge and the national immunization programme had significant association with the acceptance of HPV vaccine among their respondents (Ezat et al., 2013). Based on the results of this study, a common reason why the participants are not willing to be vaccinated is that they are not at risk of HPV infection and also they feel that the government should pay for HPV vaccination. This understanding is logical with a survey conducted earlier among women in Malaysia, which indicated approximately $80 \%$ of them are not willing to take up the vaccine as they are unable to afford for the vaccine (Liao et al., 2009). Previous reports suggested that a subsidy would reduce the self-paid price to increase the demand for vaccination, and the demand is more elastic in areas with higher prices (Kondo et al., 2012; Zhou et al., 2013). In Malaysia, the government is offering free vaccines not only to secondary school females, but to all those who are willing to be vaccinated since the year 2010 (Buang, 2010). This indicates that the participants in this study are unaware of the government schemes.

This study is a purposeful sampling of medical students from a private medical university, which limits generalizability to other populations or settings. Hence the consequences may not be representing all medical students of other universities in Malaysia. In this survey it was presumed that the hypothetical HPV vaccine was $100 \%$ efficient in preventing cervical cancer cases, which is not the current practice in the material universe.

Our study examines similarities and differences between different ethnic group (Chinese, Malay and Indian) students in a medical university on their knowledge of HPV infection, Pap smear test and HPV vaccination uptake. Importantly, we found that all three ethnic groups have higher level of knowledge and willing to recommend vaccination in future. Workshops and seminars can be conducted among the medical student community about HPV infection and cervical cancer vaccination. Awareness campaign about the role of 
government in preventing cervical cancer and subsidized vaccinations are the strategies that can be done by the academic institutions and medical universities. Private hospitals and pharmaceutical industries may go in concert with the academic establishments to further enhance the vaccine uptake by the community.

In conclusion, this study found that the knowledge of HPV infection, cervical cancer, Pap smear test, and HPV vaccination among the medical students of different ethnic groups were found high and there is no significant difference in their knowledge. University medical students represent the group of future health care providers who are able to create awareness and promote positive health behaviors towards cervical cancer prevention. Medical students had positive attitudes towards recommending HPV vaccination to the public. This group of medical students was benefited from the curriculum and clinical exposure. The prescribers strive to educate their patients on topics like HPV vaccination. Cultural differences may pose additional barriers to deal with multi ethnic group population. The medical students need high level skills on patient education and counseling. This study could serve as an indicator of the quality of future medical professionals in Malaysia to implement cervical cancer prevention programmes and policies with regards to improvement in HPV vaccination uptake, and reduce the morbidity and mortality of cervical cancer in Malaysia.

\section{Acknowledgements}

The authors of the study are grateful to all the members who participated in this study. The authors also acknowledge the support provided by Post-graduate Research Department, International Medical University, Malaysia.

\section{References}

Al-Dubai SA, Alshagga MA, Al-Naggar RA, et al (2010). Knowledge, attitudes and barriers for human papilloma virus (HPV) vaccines among Malaysian women. Asian Pac J Cancer Prev, 11, 887-92.

Aljunid S, Zafar A, Saperi S, Amrizal M (2010). Burden of disease associated with cervical cancer in Malaysia and potential costs and consequences of HPV vaccination. Asian Pac J Cancer Prev, 11, 1551-9.

Boehner CW, Howe SR, Bernstein DI, Rosenthal SL (2003). Viral sexually transmitted disease vaccine acceptability among college students. Sex Transm Dis, 30, 774-8

Chow S, Soon R, Park J, et al (2010). Knowledge, attitudes, and communication around human papillomavirus (HPV) vaccination amongst urban Asian mothers and physicians. Vaccine, 28, 3809-17.

Jenkins D (2008). A review of cross-protection against oncogenic HPV by an HPV-16/18 AS04-adjuvanted cervical cancer vaccine: importance of virological and clinical endpoints and implications for mass vaccination in cervical cancer prevention. Gynecol Oncol, 110, 18-25.

Ezat SW, Hod R, Mustafa J, et al (2013). National HPV immunization programme: knowledge and acceptance of mothers attending an obstetrics clinic at a teaching hospital, Kuala Lumpur. Asian Pac J Cancer Prev, 14, 2991-9.

Ezem BU (2007). Awareness and uptake of cervical cancer screening in Owerri, South-Eastern Nigeria. Ann Afr Med, 6, 94-8.

Dochez C, Bogers JJ, Verhelst R, Rees H (2014). HPV vaccines to prevent cervical cancer and genital warts: an update. Vaccine, 32, 1595-601.

Giede C, McFadden LL, Komonoski P, et al (2010). The acceptability of HPV vaccination among women attending the university of saskatchewan student health services. $J$ Obstet Gynaecol Can, 32, 679-86.

Holcomb B, Bailey JM, Crawford K, Ruffin MT $4^{\text {th }}$ (2004). Adults' knowledge and behaviors related to human papillomavirus infection. J Am Board Fam Pract, 17, 26-31.

Hopenhayn C, Christian A, Christian WJ, Schoenberg NE (2007). Human papillomavirus vaccine: knowledge and attitudes in two Appalachian Kentucky counties. Cancer Causes Control, 18, 627-34.

Hoque ME, Ghuman S, Hal GV (2013). Human papillomavirus vaccination acceptability among female university students in South Africa. Asian Pac J Cancer Prev, 14, 4865-9.

Jones M, Cook R (2008). Intent to receive an HPV vaccine among university men and women and implications for vaccine administration. J Am Coll Health, 57, 23-32.

Kahn JA, Cooper HP, Vadaparampil ST, et al (2009). Human papillomavirus vaccine recommendations and agreement with mandated human papillomavirus vaccination for 11-to12-year-old girls: a statewide survey of Texas physicians. Cancer Epidemiol Biomarkers Prev, 18, 2325-32.

Khoo CL, Teoh S, Rashid AK, et al (2011). Awareness of cervical cancer and HPV vaccination and its affordability among rural folks in Penang Malaysia. Asian Pac J Cancer Prev, 12, 1429-33.

Kilic A, Seven M, Guvenc G, Akyuz A, Ciftci S (2012). Acceptance of human papillomavirus vaccine by adolescent girls and their parents in Turkey. Asian Pac J Cancer Prev, 13, 4267-72.

Kondo M, Yamamura M, Hoshi SL, Okubo I (2010). Demand for pneumococcal vaccination under subsidy program for the elderly in Japan. BMC Health Serv Res, 12, 313.

Kruiroongroj S, Chaikledkaew U, Thavorncharoensap M (2014). Knowledge, acceptance, and willingness to pay for human papilloma virus (HPV) vaccination among female parents in Thailand. Asian Pac J Cancer Prev, 15, 5469-74.

Kwang NB, Yee CM, Shan LP, et al (2014). Knowledge, perception and attitude towards human papillomavirus among pre-university students in Malaysia. Asian Pac J Cancer Prev, 15, 9117-23.

La Torre G, de Waure C, Chiaradia G, Mannocci A, Ricciardi W (2007). HPV vaccine efficacy in preventing persistent cervical HPV infection: a systematic review and metaanalysis. Vaccine, 25, 8352-8.

Liao C, Liu J, Pwu R, et al (2009). Valuation of the economic benefits of human papillomavirus vaccine in Taiwan. Value Health, 12, 74-7.

Madhivanan P, Srinivas V, Marlow L, et al (2014). Indian parents prefer vaccinating their daughters against HPV at older ages. Asian Pac J Cancer Prev, 15, 107-10.

McClelland A, Liamputtong P (2006). Knowledge and acceptance of human papillomavirus vaccination: perspectives of young Australians living in Melbourne, Australia. Sex Health, 3, 95-101.

National Health Morbidity Survey III (2008). The third national health and morbidity survey. Ministry of health, Kuala Lumpur. [ONLINE] Available at: http://www.psasir.upm. edu.my/6543.

Pulcini C, Massin S, Launay O, Verger P (2013). Factors associated with vaccination for hepatitis B, pertussis, seasonal and pandemic influenza among French general 

practitioners: a 2010 survey. Vaccine, 31, 3943-9.

Rashwan H, Saat N, Manan D (2012). Knowledge, attitude and practice of malaysian medical and pharmacy students towards human papillomavirus vaccination. Asian Pac J Cancer Prev, 13, 2279-83.

Saha A, Nag Chaudhury A, Bhowmik P, et al (2010). Awareness of cervical cancer among female students of premier colleges in Kolkata, India. Asian Pac J Cancer Prev, 11, 1085-90.

Saidatul Norbaya Buang (2010), Introduction of national hpv immunization program: sharing malaysia experience presented at global meeting on cervical cancer prevention programme 29 Nov-2 Disember 2010.

Saravanan C, Kingston R, Gin M (2014). Is Test Anxiety a Problem among Medical Students: A cross sectional study on outcome of test anxiety among medical students? Int $J$ Psychol Stud, 6, 24-31.

Shafei M, Zainon N, Zulkifli N, Ibrahim M (2014). Knowledge and perception on human papilloma virus infection and vaccination among medical students of a university in Malaysia. Procedia Soc Behav Sci, 116, 2707-10.

Songthap A, Pitisuttithum P, Kaewkungwal J, et al (2009). Knowledge, attitudes, and acceptability of a human papillomavirus vaccine among healthcare providers. Southeast Asian J Trop Med Public Health, 40, 1048-56.

Uzunlar, Ozyer, Bacser E, et al (2013). A survey on human papillomavirus awareness and acceptance of vaccination among nursing students in a tertiary hospital in Ankara, Turkey. Vaccine, 31, 2191-5.

Wen Y, Pan XF, Zhao ZM, et al (2014). Knowledge of human papillomavirus (HPV) infection, cervical cancer, and HPV vaccine and its correlates among medical students in Southwest China: a multi-center cross-sectional survey. Asian Pac J Cancer Prev, 15, 5773-9.

Wong LP (2011). Knowledge and attitudes about HPV infection, HPV vaccination, and cervical cancer among rural southeast Asian women. Int J Behav Med, 18, 105-11.

Wong LP (2009). Physicians' experiences with HPV vaccine delivery: Evidence from developing country with multiethnic populations. Vaccine, 27, 1622-7.

Yilmazel G, Duman NB (2014). Knowledge, attitudes and beliefs about cervical cancer and human papilloma virus vaccination with related factors in Turkish university students. Asian Pac $J$ Cancer Prev, 15, 3699-704.

Zhou L, Su Q, Xu Z, et al (2013). Seasonal influenza vaccination coverage rate of target groups in selected cities and provinces in china by season (2009/10 to 2011/12) PLoS One, 8, 3724 . 